\title{
THE STATUS OF TEXTILE-BASED DRY EEG ELECTRODES
}

\author{
Granch Berhe Tseghai ${ }^{1,2, *}$, Benny Malengier ${ }^{1}$, Kinde Anlay Fante $^{2}$, Lieva Van Langenhove ${ }^{1}$ \\ 1 Department of Materials, Textiles and Chemical Engineering, Ghent University, Ghent, Belgium \\ 2 Jimma Institute of Technology, Jimma University, Jimma, Ethiopia \\ ${ }^{*}$ Corresponding author. E-mail: GranchBerhe.Tseghai@Ugent.be
}

\begin{abstract}
:
Electroencephalogram (EEG) is the biopotential recording of electrical signals generated by brain activity. It is useful for monitoring sleep quality and alertness, clinical applications, diagnosis, and treatment of patients with epilepsy, disease of Parkinson and other neurological disorders, as well as continuous monitoring of tiredness/ alertness in the field. We provide a review of textile-based EEG. Most of the developed textile-based EEGs remain on shelves only as published research results due to a limitation of flexibility, stickability, and washability, although the respective authors of the works reported that signals were obtained comparable to standard EEG. In addition, nearly all published works were not quantitatively compared and contrasted with conventional wet electrodes to prove feasibility for the actual application. This scenario would probably continue to give a publication credit, but does not add to the growth of the specific field, unless otherwise new integration approaches and new conductive polymer composites are evolved to make the application of textile-based EEG happen for bio-potential monitoring.
\end{abstract}

\section{Keywords:}

Electroencephalogram, brain activity monitoring, textile-based electrode

\section{Introduction}

Neurological disorders are diseases of the brain, spine, and the nerves that connect them [1]. There are more than 600 diseases of the nervous system [2], such as brain tumors, epilepsy, Parkinson's disease, stroke, and less familiar ones, like frontotemporal dementia. Medical treatment tailored to the individual patient's disorders may eventually decrease mortality and improve the quality of life; however, for this, each disorder and response to treatment must be objectively quantified. Moreover, brain disease patients do not recognize the commencement and do not perceive what is happening. Therefore, disorder detection devices that enable an objective assessment of seizure frequency and treatment tailored to the individual patient ought to be used. Fast recognition and treatment may potentially decrease morbidity and mortality through closed-loop systems. However, no single detection device can detect all diseases varieties. Therefore, the choice of a disorder detection device should consider the patientspecific disorder. For example, each type of epileptic seizure consists of one or more phenomena occurring simultaneously or sequentially. The two main components that can be evaluated to evaluate clinical features are movement and physiological signals. Movement refers to specific body parts that move in specific ways to detect the disorder, which can be identified using accelerometers [3,4], surface electromyography (sEMG) [5], video monitoring [6], or seizure-alert dogs [7]. Physiological signals include heart rate, respiratory rate, electrocardiogram (ECG) detectable sweating and temperature, exploratory data analysis (EDA) sweating, wristband temperature, and changes in respiratory rate with a thoracic band. With violent body movements and often prominent autonomous changes, a type of generalized seizure that produces bilateral, convulsive tonic and clonic muscle contractions known as generalized tonic-clonic seizures (GTCS) may occur [8]. Several types of sensors, therefore, recognize GTCS more readily than other types of seizures. On the other end of the spectrum, the absence is challenging to capture as it consists of a brief decline in awareness with minimally associated movements and is therefore often only captured by an observer or electroencephalogram (EEG).

\section{Search method}

From April 2018 to May 2019, a systematic electronic document search was conducted using the preferred reporting items for Systematic Reviews and Meta-Analyses (PRISMA) guidelines (Figure 1) of the web of science database in particular and the Google search engine in general. "Dry EEG," "wet EEG," "textile EEG electrode," "textile Electroencephalography electrode," "textile EEG sensor," "textile encephalography sensor," "textile bio-potential electrode," or "textile bio-potential sensor" have been used as keywords during searching. We did not exclude any design, measurement of results, or group of comparisons. After removing duplicates, 219 articles had been found out of 1,207 articles. Then, the articles were screened for relevance by their title and abstract seeking reference to the EEG-based textiles and 122 articles were excluded. The full text has been accessed for the remaining 97 articles. If 
they used some elements of textile technology, articles were included if the EEG was based on textiles. No research study design was specifically excluded due to the limited number of papers and the multidisciplinary nature of the research. However, if the articles were reviews and/or discussions even on textile-based EEG or if they did not use textile technology or if the primary function of the EEG textile work was simulation, they were excluded. Twenty-one articles fulfilled the criteria for inclusion in the review, of which only 9 were textile-based and the others lost their textile characteristics during electrode manufacturing. For instance, Hua et al. [9] developed a flexible multilayer semi-dry EEG electrode made of conductive composite materials with more than $3 \mathrm{~mm}$ thickness of metallic components. It is logical to say that this electrode has lost the textile nature as long as $3 \mathrm{~mm}$ metallic components are on the fabric. It should be noted that since textile-based EEGs are an emerging field of technology, researchers with commercial links may subsequently have restrictions on publishing their findings, so there may be some risk of bias in the findings.

\section{Overview of EEG electrodes}

EEG is a classic example of a biopotential recording of the electrical signals produced by the activity of the brain [9-11]. It is useful for monitoring the quality and alertness of sleep, clinical applications, diagnosis and treatment of patients with epilepsy, Parkinson's disease and other neurological disorders, and continuous monitoring of fatigue/alertness of staff deployed in the field or under strain [12]. The EEG electrodes are placed on the scalp to monitor the activities in the brain (Figure 2). EEG carries a large amount of complex information valuable for the detection of ongoing seizures [13]. As a result, EEG sensors have long been considered the gold standard for seizure diagnosis.

Several groups developed automatic seizure detection algorithms based on EEG [13-15]. Most of them used the data from the Freiburg seizure prediction and the European epilepsy database to test their algorithms using data from 2 to 6 electrodes to recreate conditions for outpatient performance.

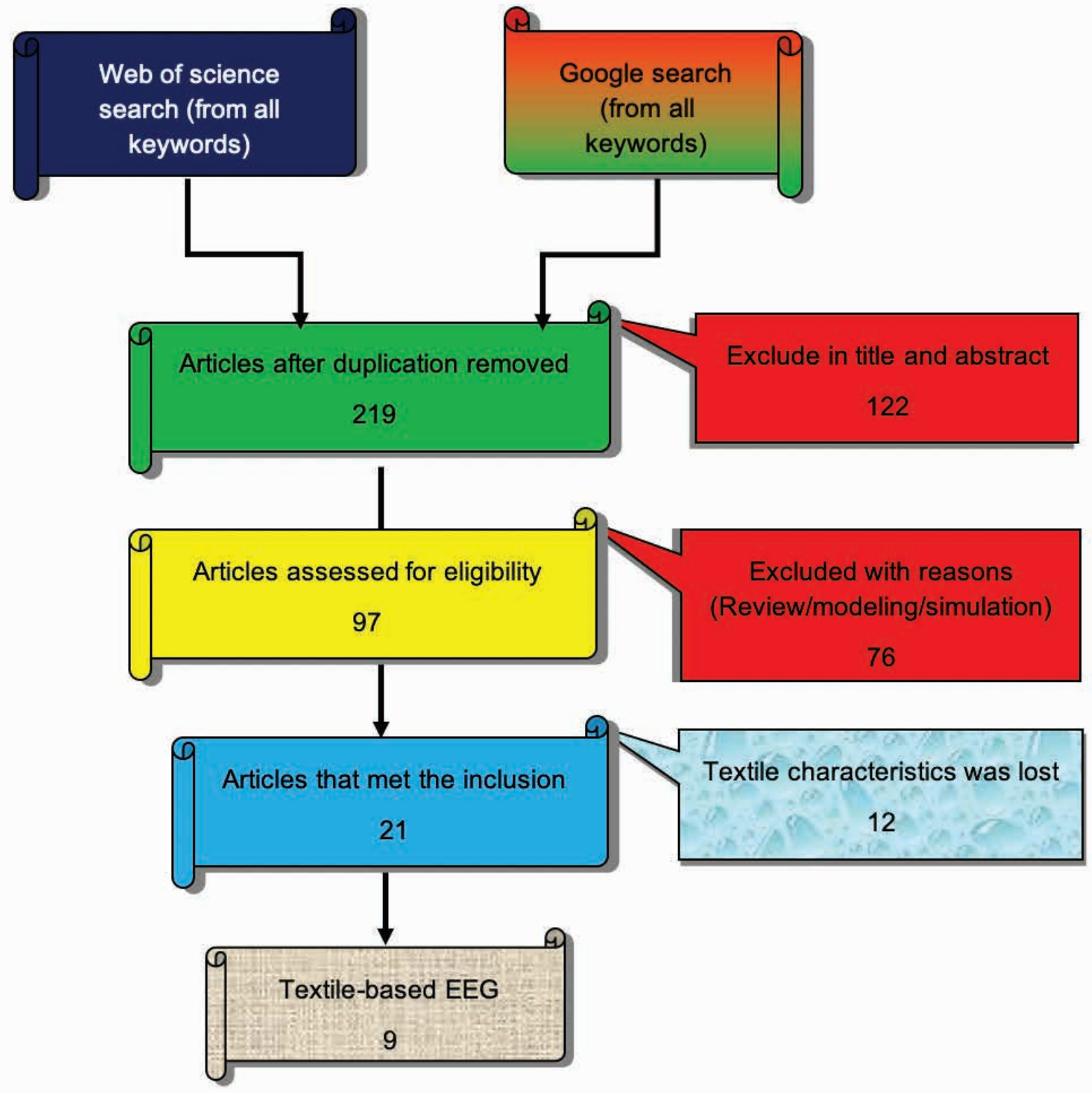

Figure 1. Flow chart of articles selection process and result according to PRISMA. 


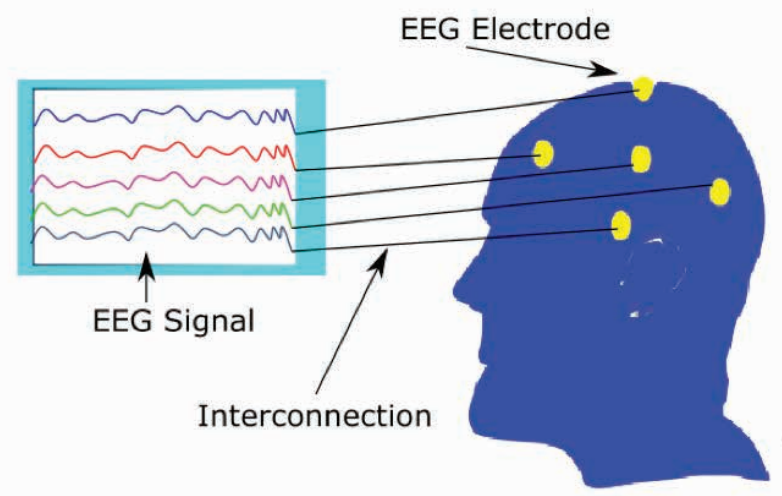

Figure 2. Brain activity measurement using EEG electrodes.

The database in Freiburg uses six electrodes: three electrodes near the focal area and three electrodes at a distance [13-16]. Furthermore, various types of novel EEG sensor electrodes have been introduced by many researchers. Chen et al. designed and developed an EEG recording frontend circuitry headband for the detection of an epileptic seizure, a textile headband with printed-circuit-board inside, and textile electrodes with a compact and low-power design for EEG recording that makes it convenient for the wearable purpose [17]. Wang et al. proposed a novel porous ceramic "semi-dry" electrode with a key feature that electrode tips can slowly and continuously release a small amount of electrolyte liquid into the scalp, providing an ionic conducting path for detecting neural signals that can effectively capture electrophysiological responses and is a viable alternative to conventional electrodes in brain-computer interface $(\mathrm{BCl})$ applications [18]. Radhakrishnan et al. developed a needle array dry electrode microstrip from stainless steel (SS) having an impedance about $6.8 \mathrm{~kW}$ at $20 \mathrm{~Hz}$ in a $0.9 \% \mathrm{NaCl}$ solution, which is sufficiently low to fulfill the requirements of biopotential measurement and suitable for penetrating the stratum corneum of the skin and acquire the EEG signal directly from the interstitial fluidic layer underneath which could make it a promising dry electrode for long duration EEG monitoring [12]. In 2017, Kannan et al. developed a wearable and less visible Ear-EEG recording

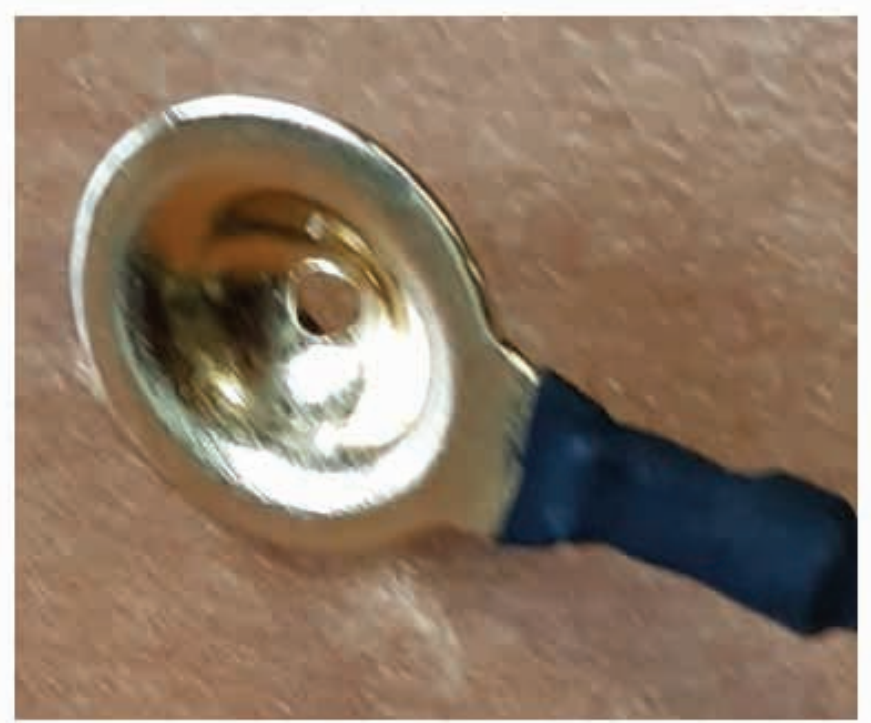

device [19]. It records Ear-EEG raw data in real-time and allows displaying the brain signals obtained, which then can be analyzed for further investigation of any particular neural disorder. Kappel et al. developed a dry-contact ear-EEG platform [20], comprising actively shielded and nanostructured electrodes embedded in an individualized soft earpiece. This electrode eliminates the gel application at the electrode-skin interface which shows its user-friendliness and technological feasibility. Xing et al. demonstrated a high performance highspeed steady-state visual evoked potentials (SSVEP)-based $\mathrm{BCl}$ system using on claw-like structure dry EEG electrodes created from thermoplastic polyurethanes (TPU) coated with conductive ink of $\mathrm{Ag} / \mathrm{AgCl}$ mixture [21]. This device can be worn comfortably over a hair-covered head area, and the pins of the electrode can go easily through the hair and contact the scalp providing a stable impedance of the electrode. Therefore, the electrode is capable to record reliable and high-quality signals for subsequent signal processing.

Moreover, commercial EEG electrodes (Figure 3) have been offered to the market in different parts of the world for research purpose, home health monitoring, and clinical purposes.

In Table 1, we provided few examples of commercially available EEG devices, such as $X$ series-EEG [22], Neuro:On Smart Sleep Mask [23], Ultracortex "Mark IV" EEG Headset [24], TGAM EEG Biosensor [25], XWave EEG [26], EEG SENSOR - T9305M [27], and SENS-EEG-UCE6 [28].

\section{Textile-based EEG electrodes}

Many dry electrodes for biopotential measurement have been researched. For instance, a textile-based electrocardiography (ECG) [28-34], electromyography (EMG) [35-39], and electrooculography (EOG) $[38,40,41]$ are among the recently reported textile-based electrodes for biopotential measurement. In this part, we only reviewed the textile-based EEG electrodes.

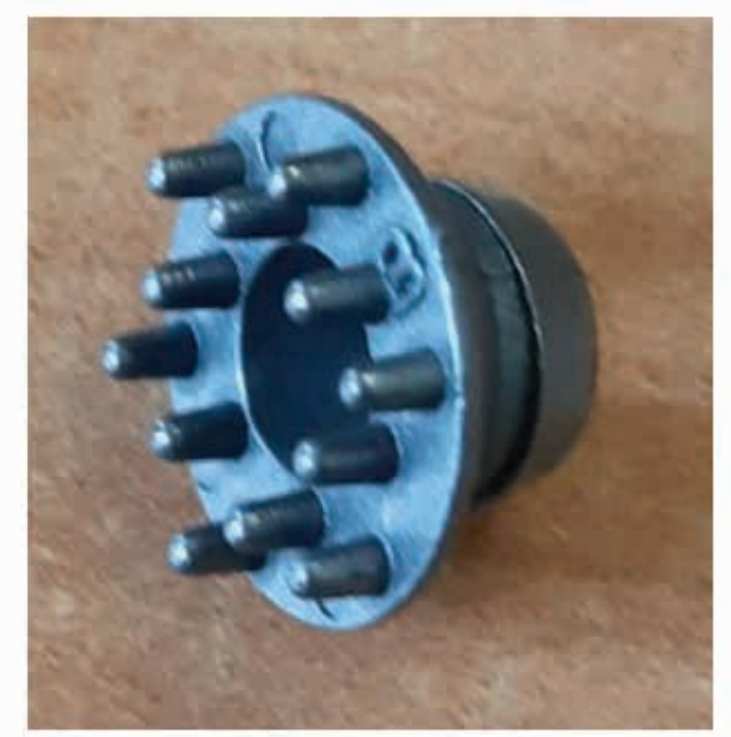

Figure 3. Standard EEG electrodes; gold cup wet electrode (left); and pin-shaped dry Ag/AgCl electrode (right). 
Table 1. Examples of the commercial EEG device

\begin{tabular}{|c|c|c|c|}
\hline Company & Brand name & Device type & Website \\
\hline $\begin{array}{l}\text { Advanced } \\
\text { brain } \\
\text { monitoring }\end{array}$ & $\begin{array}{l}X \text { series - } \\
\text { EEG wireless } \\
\text { monitoring }\end{array}$ & $\begin{array}{l}\text { EEG wireless headset for interpretation of } \\
\text { physiological signals }\end{array}$ & www.advancedbrainmonitoring.com/ \\
\hline Neuro:On & $\begin{array}{l}\text { Neuro:On Smart } \\
\text { Sleep Mask }\end{array}$ & $\begin{array}{l}\text { Measures brain waves (EEG), muscle tension } \\
\text { (EMG), eye movement (EOG), pulse (pulse } \\
\text { oximetry), body temp, and body movement } \\
\text { during sleep (actigraphy) }\end{array}$ & neuroon.com/ \\
\hline OpenBCl & $\begin{array}{c}\text { Ultracortex } \\
\text { "Mark IV" EEG } \\
\text { Headset }\end{array}$ & EEG Monitoring & openbci.com/ \\
\hline Neurosky & $\begin{array}{c}\text { ThinkGearÔ AM } \\
\text { (TGAM EEG } \\
\text { Biosensor) }\end{array}$ & Understand the mind. & neurosky.com/ \\
\hline PLX devices & XWave EEG & EEG brain-computer interface for iPhone/iPad & www.plxdevices.com/ \\
\hline $\begin{array}{l}\text { Science } \\
\text { division }\end{array}$ & $\begin{array}{l}\text { EEG SENSOR - } \\
\text { T9305M }\end{array}$ & $\begin{array}{l}\text { Provide EEG from } 2 \text { to } 1,000 \mathrm{~Hz} \text { with less than } \\
\qquad 0.3 \mathrm{mV} \text { noise }\end{array}$ & www.thoughttechnology.com/ \\
\hline Plux & $\begin{array}{l}\text { SENS-EEG- } \\
\text { UCE6 }\end{array}$ & $\begin{array}{l}\text { Purpose-built sensor for brain activity } \\
\text { measurement. }\end{array}$ & store.plux.info/ \\
\hline
\end{tabular}

EEG, electroencephalogram; EOG, electrooculography; EMG, electromyography.

There is limited information on which devices for sensor detection are optimal for each type of seizure. The ideal monitoring device for patients, families, and medical staff should be safe and easy to use. For patients, especially during sleep, it must be comfortable and therefore preferably wireless, miniaturized, and light. If electrodes are used, they must be as small and as few as possible as a significant percentage of patients are unwilling to wear long-term electrodes [13]. The device should be discreet and unobtrusive. It is important to avoid uncomfortable cables, electrodes, lights, buttons, and sounds as this disturbs the patient and family, even more so in the long run. This was confirmed by a recent survey evaluating the desires of patients, which revealed a strong preference for a seizure detection device with little interference with daily activities [42]. Using textile electrodes, therefore, overcomes the related issues and would fill the gap of existing metal electrodes. Textile electrodes are fabric-made electrodes. Textile materials are usually insulators, but the conductive yarns are attached to or incorporated in the fabric forming the textile electrodes during their production process. These electrodes need no gel to connect to the skin. Weaving, knitting, or embroidering conductive yarn to the structure can make the textile electrodes. The textile electrodes are good for long-term measurement, as they do not irritate the skin. They are also light, ductile, and washable [43].

Modern applications of EEG-based BCls rely heavily on socalled wet electrodes (e.g., $\mathrm{Ag} / \mathrm{AgCl}$ electrodes) requiring gel application and skin preparation to function properly. Standard planar/AgCl electrodes have two major drawbacks: skin preparation, which in some subjects produces abrasive lesions, and the application of conductive gel or paste, which is conductive. The patient may be sensitive to gel, which may result in an allergic reaction, rash, or irritation of the skin. Because of changes in moisture, the variation in the impedance of the gel causes artifacts for long-term monitoring. In addition, when a large number of electrodes are required, e.g. for epilepsy and Parkinson's disease monitoring, skin preparation and gel application are time-consuming. The demand for more comfortable and user-friendly electrodes has led to a growing number of dry devices being developed, which can overcome the limitations of wet electrodes. Dry electrodes have disadvantages of higher impedance of the electrodeskin boundary, additional circuitry, and sensitivity to artifacts of movement. However, it was reported in the literature that the impedance decreases considerably after a settling time due to the accumulation of perspiration under the electrodes, and the noise of artifacts is lower than that of wet electrodes [44]. Other studies have also reported that dry electrodes have several advantages over wet ones, such as the impedance of the electrode-skin interface, signal intensity, and electrode size [45].

Promising textile-based dry electrode research that provides better user comfort and stable EEG recordings has been consistently reported since 2010 from the fields of electrical, textile, material, and biomedical disciplines. In 2010, Löfuede et al. tested the EEG signals obtained with knitted soft textile EEG electrodes made of nylon, conductive fibers, spandex, and thinner yarn polypropylene [46]. It showed a larger total surface area and was very good for absorbing liquids, which could be very useful in long-term monitoring applications, particularly in neonates. In 2011, Lin et al. developed a novel dry foambased electrode EEG electrode from conductive fabric [47]. It provided partly polarizable electric characteristics, and they found better performance for long-term EEG measurement, so it could possibly be practical for daily life applications. Later in 2012, Salvo et al. fabricated 3D printed dry medical electrodes 
Table 2. Comparison of textile-based EEG electrodes with standard electrode

\begin{tabular}{|c|c|c|c|c|c|c|}
\hline No. & EEG electrode & $\begin{array}{l}\text { Conductive } \\
\text { materials } \\
\text { used }\end{array}$ & $\begin{array}{l}\text { Integration } \\
\text { approach }\end{array}$ & $\begin{array}{c}\text { Comparison with standard } \\
\text { electrode and conclusion } \\
\text { drawn }\end{array}$ & $\begin{array}{l}\text { Type of } \\
\text { comparison }\end{array}$ & Reference \\
\hline & $\begin{array}{l}\text { Nylon, conductive } \\
\text { fibers, spandex, } \\
\text { and thinner yam } \\
\text { polypropylene }\end{array}$ & $\begin{array}{l}\text { Sliver-plated } \\
\text { fibers }\end{array}$ & Knitting & $\begin{array}{l}\text { Visual inspection of EEG } \\
\text { data in time and frequency } \\
\text { domains and an EEG signal } \\
\text { was found }\end{array}$ & Not compared & [46] \\
\hline & $\begin{array}{l}\text { Conductive } \\
\text { polymer foam- } \\
\text { conductive fabric }\end{array}$ & $\mathrm{Ni} / \mathrm{Cu}$ & Coating & $\begin{array}{l}\text { Not compared with the } \\
\text { standard electrode, but an } \\
\text { EEG signal was found }\end{array}$ & Qualitative & [47] \\
\hline & $\begin{array}{l}\text { 3D conductive } \\
\text { acrylic } \\
\text { photopolymer }\end{array}$ & $\begin{array}{l}\text { Titanium and } \\
\text { gold }\end{array}$ & 3D printing & $\begin{array}{c}\text { Visual observation of EEG } \\
\text { signal with wet electrodes } \\
\text { and the comparable result } \\
\text { was found }\end{array}$ & Qualitative & [48] \\
\hline & $\begin{array}{c}\text { Knitted } \\
\text { conductive fabric }\end{array}$ & $\begin{array}{l}\text { Silver-plated } \\
\text { conductive } \\
\text { fibers }\end{array}$ & Knitting & $\begin{array}{l}\text { Visual inspection of EEG } \\
\text { data in time and frequency } \\
\text { domains with standard } \\
\text { electrodes and found } \\
\text { comparable results }\end{array}$ & Qualitative & [49] \\
\hline & $\begin{array}{l}\text { Copper plated } \\
\text { polyester fabrics }\end{array}$ & Copper & $\begin{array}{l}\text { Electroless } \\
\text { copper plating }\end{array}$ & $\begin{array}{l}\text { Compared with commercially } \\
\text { available electrodes and } \\
\text { similar signal was found }\end{array}$ & Qualitative & [50] \\
\hline & $\begin{array}{l}\text { Nanostructures } \\
\text { sensor electrode } \\
\text { array }\end{array}$ & Not disclosed & $\begin{array}{l}\text { Seamlessly } \\
\text { integration }\end{array}$ & $\begin{array}{l}\text { Not compared with the } \\
\text { standard electrode but a } \\
\text { mu-wave attenuation was } \\
\text { detected }\end{array}$ & Not compared & [51] \\
\hline & $\begin{array}{l}\text { Polyaniline- } \\
\text { coated } \\
\text { polyurethane } \\
\text { foam }\end{array}$ & Polyaniline & Coating & $\begin{array}{l}\text { Visual comparison of } \\
\text { EEG data with standard } \\
\text { electrodes }\end{array}$ & Qualitative & [52] \\
\hline & $\begin{array}{l}\text { Ti and PDMS } \\
\text { foam }\end{array}$ & $\begin{array}{l}\text { Porous } \\
\text { titanium }\end{array}$ & Leaching & $\begin{array}{l}\text { Not compared with the } \\
\text { standard electrode, but an } \\
\text { EEG signal was found }\end{array}$ & Not compared & [53] \\
\hline & $\begin{array}{l}\text { Pin-shaped } \\
\text { carbon fiber }\end{array}$ & Carbon fiber & Coating & $\begin{array}{l}\text { Not compared with standard } \\
\text { electrode, but an EEG signal } \\
\text { was found }\end{array}$ & Not compared & [54] \\
\hline
\end{tabular}

by sputtering titanium as an adhesion promotion layer, evaporating gold to lower the impedance and prevent oxidation of the electrode and finally insulating it with an acrylic-based photopolymer [48]. They found qualitative results comparable to wet electrodes. In the same year, Löfhede et al. developed a long-term EEG electrode for newborns based on several electrodes distributed over the head of the baby [49]. A test on five healthy adults was performed and was found comparable to standard quality electrodes. Researchers working in the field have consistently been looking for an EEG electrode with better flexibility and stable signals. In 2014, Kumar and Thilagavathi created a copper-plated polyester fabric for EEG measurement and found similar signals as with commercially available electrodes hinting at their feasible long-term EEG monitoring candidacy [50]. In 2015, Sahi et al. worked on the development of a textile-based nanostructure sensor electrode array for EEG measurement in the motor cortex and the occipital lobe for autism disorder. A mu-wave attenuation was detected [51]. In 2016, the development of EEG electrodes from different conductive textile materials has resulted in several research results. Muthukumar et al. developed a polyaniline (PANI) polymer-coated polyurethane $(\mathrm{PU})$ foam textile electrode with surface resistance and impedance values of $7 \mathrm{~kW} /$ square and $1.45 \mathrm{MW} / \mathrm{square}$, resulting in similar results to commercial Ag/ $\mathrm{AgCl}$ electrodes, making it feasible for EEG measurements, especially for continuous monitoring purposes [52]. Peng et al. presented a skin-electrode with relatively low contact impedance based on porous titanium (Ti) for EEG recording that does not cause skin irritations or allergic reactions [53]. In 2018, Gao et al. introduced a novel soft pin-shaped dry electrode fabricated from carbon fiber bristles for EEG reading [54]. The carbon fibers were processed to reduce the contact impedance between the skin and dry electrode and realize a larger contact area and better comfort with a smaller pressure.

Apart from the development of textile-based electrodes, researchers have also been working on reviewing, testing, and characterizing existing electrodes and searching for better EEG monitoring software. Renz et al. reported a review of the recent progress in interfacing both the central and peripheral nervous 
systems for long-term functional devices [55]. The review could serve as a guide for long-term functional electrodes interfacing neural tissue. Another deep review work of Zerafa et al. highlighted the strengths and weaknesses of the three categories of steady-state visually evoked potential training methods [56]. Feature extraction techniques incorporating certain training data address and have in fact outperformed training-free methods: subject-specific $\mathrm{BCls}$ are tailored to the individual, delivering the best performance at the cost of long, and tiring training sessions. These do make these methods unsuitable for one time or sporadic use, creating a remaining need for subject-independent BCls. Senn et al. suggested a single-source multipolar stimulation (SSMPS), a novel form of stimulation based on a single current supply and a passive present day divider [57]. This demonstrates that the SSMPS efficiently limits the broadening of the excitatory field along with the electrode array and subsequently reduces the neural excitation. Craik et al. summarized the present-day practices and performance effects in the use of EEG [58]. This EEG classification provides sensible guidelines for selecting many hyper-parameters in the hope of promoting or informing the deployment of deep learning to EEG datasets in future research. Sadatnejad et al. studied the EEG representation using a multi-instance framework on the manifold of symmetrically effective precise matrices by using computerized attenuation of the extra-physiologic noise contribution and exploiting the discriminative statistics of physiological artifacts [59].

\section{Outlook on the textile-based EEG electrodes}

The conclusions drawn in the published works showed that the developed textile-based dry EEG electrodes gave similar and comparable EEG signals when compared with the commercially available $\mathrm{Ag} / \mathrm{AgCl}$ wet electrodes. Although promising comparisons were made, the findings in each research work were not compared with standard electrodes in quantitative and figurative terms, but merely referred to in qualitative terms. Therefore, to bring such electrodes into practical real-world applications, scientific research that could show the actual values of textile EEG electrodes versus standard electrodes must be conducted. Otherwise, publishing-based research would likely continue to lead to a publication but will not allow other researchers to determine what is really important, as any conductive material can be used to create an electrode. In Table 2, we presented the method of comparison used for the textile-based EEG electrode signal.

Table 2 shows that not all the textile-based EEG electrodes were quantitatively compared with standard electrodes. Moreover, there is no information on flexibility, tensile strength, thickness, and other physical characteristics of the electrode to determine if using textiles had benefited over standard electrodes. We suggest such properties of the textile should also be characterized as long as we are dealing with textilebased electrodes.

\section{Conclusion}

Existing EEG devices rely on wet electrodes (e.g., Ag/AgCl electrodes) with three major drawbacks: abrasive lesions during skin preparation, allergic reaction due to the use of the conductive gel or paste, and artifacts due to moisture change. In addition, when a large number of electrodes are required, skin preparation and gel application are time-consuming. The demand for more comfortable and user-friendly electrodes has led to the development of an increasing number of dry devices capable of overcoming the limitations of wet electrodes, but dry electrodes have the disadvantages of higher electrodeskin impedance, additional circuitry, and movement artifact susceptibility. However, it was reported in the literature that the impedance decreases considerably after a settling time due to the accumulation of perspiration under the electrodes, and the noise of artifacts is reported to be lower than that of wet electrodes.

The ideal monitoring device for patients, families, and medical staff should be safe and easy to use. For patients, especially during sleep, it must be comfortable and therefore preferably wireless, miniaturized, and light. It is important to avoid uncomfortable cables, electrodes, lights, buttons, and sounds as this disturbs the patient and family, even more so in the long run. Textile electrodes are a logical next step in the development of electrodes. These electrodes need no gel to connect to the skin. It is possible to make the textile electrodes by weaving, knitting, or embroidering conductive yarn to the structure or coating, and printing of conductive pastes on the surface of the substrate. In addition, different material types can be deposited on the textile fabric layer by layer at different paste viscosity allowing the possibility of covering electronic components vulnerable to top layer washing using waterinsoluble hydrophobic polymers. Most of the so far developed and published textile-based EEGs are kept on shelves only due to limited flexibility, skin adhesiveness, and washability. Therefore, new approaches to integration and new conductive polymer composites should be evolved to overcome the associated limitations and to make the application of textilebased EEG for biopotential monitoring a reality. At the same time, reporting on textile-based dry-electrodes should always include a discussion on the textile properties of the electrodes, and a quantitative comparison of the EEG signal with standard wet electrodes.

\section{Acknowledgment}

The authors would like to express appreciation for the support of the NASCERE and Erasmus+ project Skills4Smartex. The NASCERE project was sponsored with funds from the Ethiopian Government and the Erasmus+ project Skills4Smartex has been funded with support from the European Commission. Granch Berhe Tseghai was funded by NASCERE and Benny Malengier was supported by Erasmus+ project Skills4Smartex. 


\section{References}

[1] Chetna, M., Shah, S. (2008). Diseases of the brain and nervous system caution. Team Spirit (India) Pvt. Ltd (Ahmedabad).

[2] Matilla-dueñas, A., Corral-juan, M. (2017). Rare diseases epidemiology: update and overview, vol. 1031, Springer International Publishing.

[3] Beniczky, S., Polster, T., Kjaer, T. W., Hjalgrim, H. (2013). Detection of generalized tonic-clonic seizures by a wireless wrist accelerometer: A prospective, multicenter study. Epilepsia, 54(4), 1-4.

[4] Kusmakar, S., Karmakar, C. K., Yan, B., O’Brien, T. J., Muthuganapathy, R., Palaniswami, M. (2019). Automated detection of convulsive seizures using a wearable accelerometer device. IEEE Transactions on Biomedical Engineering, 66(2), 421-432.

[5] Halford, J. J., Sperling, M. R., Nair, D. R., Dlugos, D. J., Tatum, W. O., et al. (2017). Detection of generalized tonic-clonic seizures using surface electromyographic monitoring. Epilepsia, 58(11), 1861-1869.

[6] Lee, J. W. (2018). Real-time non-EEG convulsive seizure detection devices: they work; now what? Epilepsy Currents, 18(3), 164-166.

[7] Strong, V., Brown, S. W., Walker, R. (1999). Seizure-alert dogs - fact or fiction? Seizures, 8, 62-65.

[8] Miller, J. W. (2010). Are generalized tonic-clonic seizures really 'generalized'? Epilepsy Currents, 10(4), 80-81.

[9] Hua, H., Tang, W., Xu, X., Feng, D. D., Shu, L. (2019). Flexible multi-layer semi-dry electrode for scalp EEG measurements at Hairy sites. Micromachines, 10(518), 1-13.

[10] Sun, Y., Lo, F. P. W., Lo, B. (2019). EEG-based user identification system using $1 D$-convolutional long shortterm memory neural networks. Expert System with Applications, 125, 259-267.

[11] Khalaf, A., Sejdic, E., Akcakaya, M. (2019). EEG-fTCD Hybrid brain-computer interface using template matching and wavelet decomposition. Journal of Neural Engineering, 16(3).

[12] Radhakrishnan, J. K., Nithila, S., Kartik, S. N., Bhuvana, T., Kulkarni, G. U., et al. (2018). A novel, needlearray dry-electrode with stainless steel micro-tips, for electroencephalography monitoring. Journal of Medical Device, 12(4), 1-7.

[13] Wunder, S., Hunold, A., Fiedler, P., Schlegelmilch, F., Schellhorn, K., et al. (2018). Novel bifunctional cap for simultaneous electroencephalography and transcranial electrical stimulation. Science Reports, 8(1), 1-11.

[14] Alawieh, H., Hammoud, H., Haidar, M., Nassralla, M. H., El-Hajj, A. M., et al. (2016). Patient-aware adaptive ngrambased algorithm for epileptic seizure prediction using EEG signals. 2016 IEEE 18th International Conference on e-Health Networking, Application and Services Healthcom, 2016, 1-6.

[15] Spies, R., Gassert, R. (2019). A penalized time-frequency band feature selection and classification procedure for improved motor intention decoding in multichannel EEG. Journal of Neural Engineering, 16(1), 016019.
[16] Al Ghayab, H. R., Li, Y., Siuly, S., Abdulla, S. (2018). Epileptic seizures detection in EEGs blending frequency domain with information gain technique. Soft Computing, 23(1), 227-239.

[17] Chen, Y. J., Lin, Y. S., Chiueh, H. (2016). EEG recording frontend circuitry for epileptic seizure detection headband. 2016 IEEE Healthcare Innovations and Point-of-Care Technologies, Conference HI-POCT, 2016, 42-45.

[18] Wang, F., Li, G., Chen, J., Duan, Y. (2016). Novel semidry electrodes for brain-computer interface applications. Journal of Neural Engineering, 13(4), 046021.

[19] Kannan, R., Ali, S. S. A., Farah, A., Adil, S. H., Khan, A. (2017). Smart wearable EEG sensor. Procedia Computer Science, 105(December 2016), 138-143.

[20] Kappel, S. L., Rank, M. L., Toft, H. O., Andersen, M., Kidmose, P. (2018). Dry-contact electrode ear-EEG. IEEE Transactions on Biomedical Engineering, 66(1), 150-158.

[21] Xing, X., Wang, Y., Pei, W., Guo, X., Liu, Z., et al. (2018). A high-speed SSVEP-based BCl using dry EEG electrodes. Scientific Reports, 8(1), 1-10.

[22] Advanced Brain Monitoring. (2019). X series - EEG wireless monitoring. [Online]. Web site: https://www. advancedbrainmonitoring.com/.

[23] Neuro:On. (2019). Neuro: on smart sleep mask. [Online]. Web site: https://neuroonopen.com/.

[24] OpenBCl. (2019). Ultracortex 'mark IV' EEG headset. [Online]. Web site: https://openbci.com/.

[25] Neurosky. (2019). ThinkGear ${ }^{T M}$ AM (TGAM EEG biosensor). [Online]. Web site: http://neurosky.com/ biosensors/eeg-sensor/.

[26] PLX Devices. (2019). XWave EEG. [Online]. Web site: https://www.plxdevices.com/.

[27] Science Division. (2019). EEG SENSOR - T9305M. [Online]. Web site: http://www.thoughttechnology.com/ sciencedivision/pages/products/eegflex.html.

[28] Plux. (2019). SENS-EEG-UCE6. [Online]. Web site: https:// plux.info/.

[29] Lee, E., Cho, G. (2019). PU nanoweb-based textile electrode treated with single-walled carbon nanotube/ silver nanowire and its application to ECG monitoring. Smart Materials and Structures, 28(4), 045004.

[30] Ankhili, A., Tao, X., Koncar, V., Coulon, D., Tarlet, J. (2019). Ambulatory evaluation of ECG signals obtained using washable textile-based electrodes made with. Sensors, 19(416), 13.

[31] Kang, T., Park, J., Yun, G., Hee, H., Lee, H. (2019). A realtime humidity sensor based on a microwave oscillator with conducting polymer PEDOT : PSS film. Sensors Actuators B. Chemical, 282, 145-151.

[32] Achilli, A., Bonfiglio, A., Pani, D. (2018). Design and characterization of screen-printed textile electrodes for ECG monitoring. IEEE Sensors Journal, 18(10), 40974107.

[33] An, X., Stylios, G. K. (2018). A hybrid textile electrode for electrocardiogram (ECG) measurement and motion tracking. Materials (Basel), 11(10), pii: E1887.

[34] Wu, W., Pirbhulal, S., Sangaiah, A. K., Mukhopadhyay, S. C., Li, G. (2018). Optimization of signal quality over comfortability of textile electrodes for ECG monitoring in fog computing based medical applications. Future Generation Computer Systems, 86, 515-526. 
[35] Das, P. S., Park, J. Y. (2017). A flexible touch sensor based on conductive elastomer for biopotential monitoring applications. Biomedical Signal Processing Control, 33, 72-82.

[36] Lee, S., Kim, M. O., Kang, T., Park, J., Choi, Y. (2018). Knit band sensor for myoelectric control of surface EMG-based prosthetic hand. IEEE Sensors Journal, 18(20), 85788586.

[37] Shafti, A., Ribas Manero, R. B., Borg, A. M., Althoefer, K., Howard, M. J. (2017). Embroidered electromyography: a systematic design guide. IEEE Transactions on Neural Systems and Rehabilitation Engineering, 25(9), 14721480.

[38] Yand, G., Deng, J., Pang, G., Zhang, H., Li, J., et al. (2018). An loT-enabled stroke rehabilitation system based on smart wearable armband and machine learning. IEEE Journal of Translational Engineering in Health and Medicine, 6(March, 2018).

[39] Paul, G. M., Cao, F., Torah, R., Yang, K., Beeby, S., et al. (2014). A smart textile based facial EMG and EOG computer interface. IEEE Sensors Journal, 14(2), 393400.

[40] Niijima, A., Isezaki, T., Aoki, R., Watanabe, T. (2017). hitoeCap: wearable EMG sensorfor monitoring masticatory muscles with PEDOT-PSS textile electrodes. In: ISWC '17 Proceedings of the 2017 ACM International Symposium on Wearable Computers, Maui, Hawaii. September 11-15, 2017, 215-220.

[41] Kang, T. H., Merritt, C., Karaguzel, B., Wilson, J., Franzon, $P$., et al. (2006). Sensors on textile substrates for homebased healthcare monitoring. Conference Proceedings 1st Transdisciplinary Conference on Distributed Diagnosis Home Healthcare, D2H2 2006, vol. 2006, Arlington, VA, USA, 2-4 April 2006, 5-7.

[42] Wu, J., Jia, W., Xu, C., Gao, D., Sun, M. (2017). Impedance analysis of ZnO nanowire coated dry EEG electrodes. Journal of Biomedical Engineering Informatics, 3(1), 44.

[43]. Li, H., Chen, X., Cao, L., Zhang, C., Tang, C., et al. (2017). Textile-based ECG acquisition system with capacitively coupled electrodes. Transactions of the Institute of Measurement and Control, 39(2), 141-148.

[44] Kirkup, L., Searle, A. (2000). A direct comparison of wet, dry and insulating bioelectric recording electrodes. Physiological Measurement, 21(2), 271-283.

[45] Lopez-Gordo, M. A., Sanchez Morillo, D., Pelayo Valle, F. (2014). Dry EEG electrodes. Sensors (Switzerland), 14(7), 12847-12870.

[46] Löfuede, J., Seoane, F., Thordstein, M. (2010). Soft textile electrodes for EEG monitoring. In: Proceedings of the IEEE/EMBS Region 8 International Conference on Information Technology Applications in Biomedicine, ITAB, Corfu, Greece, 3-5 November 2010, 4-7.

[47] Lin, C.-T., Liao, L.-D., Liu, Y.-H., Wang, I.-J., Lin, B.-S., et al. (2011). Novel dry polymer foam electrodes for long-term EEG measurement. IEEE Transactions on Biomedical Engineering, 58(5), 1200-1207.
[48] Salvo, P., Raedt, R., Carrette, E., Schaubroeck, D., Vanfleteren, J., et al. (2012). A 3D printed dry electrode for ECG/EEG recording. Sensors Actuators, A: Physical, 174(1), 96-102.

[49] Löfhede, J., Seoane, F., Thordstein, M. (2012). Textile electrodes for EEG recording - a pilot study. Sensors (Switzerland), 12(12), 16907-16919.

[50] Kumar, N. M., Thilagavathi, G. (2014). Design and development of textile electrodes for EEG measurement using copper plated polyester fabrics. Journal of Textile and Apparel, Technology and Management, 8(4), 1-8.

[51] Sahi, A., Rai, P., Oh, S., Ramasamy, M., Harbaugh, R. E., et al. (2014). Neural activity based biofeedback therapy for Autism spectrum disorder through wearable wireless textile EEG monitoring system. Nanosensors, Biosensors, Info-Tech Sensors Systems, 9060, 1-9.

[52] Muthukumar, N., Thilagavathi, G., Kannaian, T. (2016). Polyaniline-coated foam electrodes for electroencephalography (EEG) measurement. The Journal of Textile Institute, 107(3), 283-290.

[53] Peng, H. L., Liu, J.-Q., Tian, H.-G., Dong, Y.-Z., Yang, B., et al. (2016). A novel passive electrode based on porous Ti for EEG recording. Sensors Actuators, B Chem., 226, 349-356.

[54] Gao, K. P., Yang, H. J., Wang, X. L., Yang, B., Liu, J. Q. (2018). Soft pin-shaped dry electrode with bristles for EEG signal measurements. Sensors Actuators, A Physical, 283, 348-361.

[55] Renz, A. F., Reichmuth, A. M., Stauffer, F., ThompsonSteckel, G., Janos, V. (2018). A guide towards long-term functional electrodes interfacing neuronal tissue. Journal of Neural Engineering, 15(6), 061001.

[56] Zerafa, R., Camilleri, T., Falzon, O., Camilleri, K. P. (2018). To train or not to train? A survey on training of feature extraction methods for SSVEP-based BC/s. Journal of Neural Engineering, 15(5), 051001.

[57] Senn, P., Shepherd, R. K., Fallon, J. B. (2018). Focused electrical stimulation using a single current source. Journal of Neural Engineering, 15(5), 056018.

[58] Craik, A., He, Y., Contreras-Vidal, J. L. (2019). Deep learning for electroencephalogram (EEG) classification tasks: A review. Journal of Neural Engineering, 16(3), 031001.

[59] Sadatnejad, K., Rahmati, M., Rostami, R. (2019). EEG representation using multi-instance framework on the manifold of symmetric positive definite matrices. Journal of Neural Engineering, 16(3), 036016.

[60]Zhang, X., D'Arcy, R., Menon, C. (2019). Scoring upper-extremity motor function from EEG with artificial neural networks: a preliminary study. Journal of Neural Engineering, 16(3), 036013.

[61] Higgins, G., Faul, S., Glavin, M., Jones, E., McGinley, B., et al. (2013). The effects of lossy compression on diagnostically relevant seizure information in EEG signals. IEEE Journal of Biomedical Health Informatics, 17(1), 121 127.

[62] Golparvar, A. J., Yapici, M. K. (2018). Electrooculography by wearable graphene textiles. IEEE Sensors Journal, 18(21), 8971-8978. 